OPEN ACCESS

Edited by:

Richard Ivell,

University of Nottingham,

United Kingdom

Reviewed by:

Alex C. Varghese,

Astra Fertility Group, Canada

Yongrui Du,

Second Hospital of Tianjin Medical

University, China

${ }^{*}$ Correspondence:

Ying Wang

wy1987@ustc.edu.cn

Bo Xu

bio_xubo@163.com

Specialty section: This article was submitted to

Reproduction,

a section of the journal

Frontiers in Endocrinology

Received: 21 July 2021 Accepted: 15 October 2021 Published: 02 November 2021

Citation:

Liu R, Bai S, Jiang $X$, Luo L, Tong $X$, Zheng S, Wang Y and Xu B (2021)

Multifactor Prediction of Embryo

Transfer Outcomes Based on a Machine Learning Algorithm.

Front. Endocrinol. 12:745039. doi: 10.3389/fendo.2021.745039

\section{Multifactor Prediction of Embryo Transfer Outcomes Based on a Machine Learning Algorithm}

\author{
Ran Liu ${ }^{1}$, Shun Bai ${ }^{1}$, Xiaohua Jiang ${ }^{1}$, Lihua Luo ${ }^{1}$, Xianhong Tong ${ }^{1}$, Shengxia Zheng ${ }^{1}$, \\ Ying Wang ${ }^{2 *}$ and $\mathrm{Bo} \mathrm{Xu}^{1 *}$
}

\footnotetext{
${ }^{1}$ Reproductive and Genetic Hospital, The First Affiliated Hospital of USTC, Division of Life Sciences and Medicine, University of Science and Technology of China, Hefei, China, ${ }^{2}$ Department of Neurosurgery, The First Affiliated Hospital of USTC, Division of Life Sciences and Medicine, University of Science and Technology of China, Hefei, China
}

In vitro fertilization-embryo transfer (IVF-ET) technology make it possible for infertile couples to conceive a baby successfully. Nevertheless, IVF-ET does not guarantee success. Frozen embryo transfer (FET) is an important supplement to IVF-ET. Many factors are correlated with the outcome of FET which is unpredictable. Machine learning is a field of study that predict various outcomes by defining data attributes and using relevant data and calculation algorithms. Machine learning algorithm has been widely used in clinical research. The present study focuses on making predictions of early pregnancy outcomes in FET through clinical characters, including age, body mass index (BMI), endometrial thickness (EMT) on the day of progesterone treatment, good-quality embryo rate (GQR), and type of infertility (primary or secondary), serum estradiol level (E2) on the day of embryo transfer, and serum progesterone level $(\mathrm{P})$ on the day of embryo transfer. We applied four representative machine learning algorithms, including logistic regression $(\mathrm{LR})$, conditional inference tree, random forest (RF) and support vector machine (SVM) to build prediction models and identify the predictive factors. We found no significant difference among the models in the sensitivity, specificity, positive predictive rate, negative predictive rate or accuracy in predicting the pregnancy outcome of FET. For example, the positive/negative predictive rate of the SVM (gamma $=1$, cost $=100,10$-fold cross validation) is 0.56 and 0.55 . This approach could provide a reference for couples considering FET. The prediction accuracy of the present study is limited, which suggests that there may be some other more effective predictors to be developed in future work.

Keywords: machine learning, embryo transfer, hormone replacement cycle, good-quality embryo ratio, endometrial thickness 


\section{INTRODUCTION}

Frozen embryo transfer (FET) can not only avoid the occurrence of ovarian hyperstimulation syndrome but also avoid the adverse effects of superphysiological estrogen and early elevated progesterone on embryo implantation (1). However, it is difficult to predict the success rate objectively during the FET cycle (2). Failure or abortion of embryo transfer (ET) places psychological and economic burdens on the couple. Early knowledge of the outcome can relieve infertile couples from experiencing serious psychological stress and help them develop more reasonable expectations (3). Therefore, identifying factors that can accurately predict the success rate would be clinically significant.

A large number of studies have found that endometrial receptivity is one of the main factors affecting the pregnancy outcome. Appropriate estrogen and progesterone levels and their periodic changes are the key factors that regulate the receptive state of the endometrium. Endometrial thickness (EMT) and morphology are also closely related to endometrial receptivity (4). Yuval found that EMT $>7 \mathrm{~mm}$ was one of the necessary conditions for successful pregnancy (5). In addition, type A endometrium has a higher clinical pregnancy rate than type B and C endometrium (6). However, Golbasi et al. did not demonstrate any significant relationship between EMT changes and clinical pregnancy rates during FET cycles (7). The endometrial morphology is subjectively judged by a sonographer, so the comparability is poor. Some clinicians do not believe that endometrial morphology should be used as an index to evaluate endometrial receptivity. In addition, the quality of the embryo and maternal age are also major factors affecting pregnancy outcomes. Until now, it is still uncertain which factors have the best ability to predict pregnancy outcomes during hormone replacement FET cycles.

Machine learning algorithms have been applied in the field of assisted reproduction. Liu et al. established and compared six classification models that can accurately predict early pregnancy loss. They also found that the random forest (RF) model has the highest predictive ability. However, the patients in the study underwent fresh ET, and the predicted result was early pregnancy loss (8). Xi et al. proposed that artificial intelligence (AI) based on determinant-weighting analysis could provide an individualized embryo selection strategy for any given patient and predict the clinical pregnancy rate and twin risk (9). However, there are few studies using clinical characters on the day of ET to predict the early pregnancy outcomes of patients undergoing hormone replacement FET cycles through machine learning algorithms.

We applied four machine learning algorithms, including logistic regression (LR), conditional inference tree, RF, and support vector machine (SVM), to select features and establish prediction models. Finally, we compared above models to predict the outcome of FET (i.e., success or failure of a clinical pregnancy).

\section{MATERIALS AND METHODS}

\section{Data Sources}

In this study, 401 patients who underwent hormone replacement FET cycles were enrolled in the First Affiliated Hospital of
University of Science and Technology of China (USTC) from December 2019 to August 2020. Data included age (the day when oocytes were picked up), body mass index (BMI), type of infertility (primary or secondary), endometrial preparation protocol (gonadotropin releasing hormone-agonist down regulation or non gonadotropin releasing hormone-agonist down regulation), type of transferred embryo (cleaved embryo or blastocyst), number of transferred embryos per transferred cycle, number of good-quality embryos per transferred cycle, good-quality embryo rate (GQR), serum estrogen level (E2) on the day of ET, serum progesterone level $(\mathrm{P})$ on the day of ET, EMT and endometrial morphology (type A or type B) on the day of progesterone treatment. The interval between the day of FET and oocyte picking-up was no more than six months. The clinical characters of the two groups are shown in Table 1.

\section{Endometrial Preparation and ET}

From the second to third day of the menstrual period or withdrawal bleeding, the patients were given oral estradiol valerate $6 \mathrm{mg} /$ day. After 8-12 days, according to the vaginal Bultrasound monitoring of EMT, the hormone regimen was appropriately adjusted. If the endometrial growth was not ideal, we then added progesterone/estradiol (Femoston) red tablets containing $1 \mathrm{mg}$ vaginal medication. When the EMT of the patients was greater than or equal to $8 \mathrm{~mm}$, progesterone was added to transform the endometrium. For patients with day 3 cleavage stage embryos, ET was performed on the fourth day after progesterone was added. For patients with blastocysts, ET was performed on the sixth day after progesterone was added. The same doses of estrogen and progesterone were continued until obtaining a serum $\beta$ - human chorionic gonadotropin assay 14 or 12 days after ET. If the pregnancy test was positive, hormone replacement continued for another 8 weeks, and the patients were followed with serial ultrasonography to determine fetal viability.

\section{Endometrial Morphology}

The endometrium was divided into three types according to Gonen classification criteria (10). Type A: typical trilinear or multilayered endometrium with strong gyrus in the outer and central layers. The area between the outer layer and the midline of uterine cavity is hypoechoic or anechoic. Type B: homogeneous moderate echo, strong echo in uterine cavity and unclear midline. Type C: homogeneous hyperechoic without midline echo.

\section{Embryo Score}

Cleaved embryo score: The cleaved embryos were graded according to the number and shape of blastomeres, cytoplasmic granules and cytoplasmic fragments. The classification criteria are mainly based on Istanbul consensus (11). Grade I: the size of the blastomeres is uniform, the shape is regular, and the fragments are less than 10\%; Grade II: the blastomeres are slightly uneven or irregular, the cytoplasm has granules, and the fragments are between $10 \%$ and $20 \%$; Grade III: the blastomeres are obviously uneven or irregular, the cytoplasm has granules, and the fragments are between $20 \%$ and $50 \%$; 
TABLE 1 | The clinical characters of the patients.

\begin{tabular}{|c|c|c|c|c|}
\hline Clinical characteristics & $\begin{array}{c}\text { Total } \\
(n=401)\end{array}$ & $\begin{array}{l}\text { clinical pregnancy } \\
\qquad(\mathrm{n}=204)\end{array}$ & $\begin{array}{l}\text { Non-clinical pregnancy } \\
\qquad(\mathrm{n}=197)\end{array}$ & $\mathbf{P}$ \\
\hline Age (year), mean \pm s.d. & $31.7 \pm 5.3$ & $30.7 \pm 4.8$ & $32.7 \pm 5.6$ & 0.0002 \\
\hline BMl $\left(\mathrm{kg} / \mathrm{m}^{2}\right)$, mean $\pm \mathrm{s} . \mathrm{d}$ & $22.6 \pm 3.0$ & $22.7 \pm 3.4$ & $22.4 \pm 2.6$ & 0.37 \\
\hline Type of infertility, n (\%) & & & & 0.01 \\
\hline Primary & $205(51.1)$ & $117(57.4)$ & $88(44.7)$ & \\
\hline Secondary & 196 (48.9) & $87(42.7)$ & 109 (55.3) & \\
\hline Endometrial preparation protocol, n (\%) & & & & 0.19 \\
\hline $\mathrm{GnRH}-\mathrm{a}-\mathrm{HRT}$ & $116(28.9)$ & $65(31.9)$ & $51(25.9)$ & \\
\hline HRT & $285(71.1)$ & $139(68.1)$ & $146(74.1)$ & \\
\hline Number of transferred embryos, n (\%) & & & & 0.47 \\
\hline 1 & $184(45.9)$ & $90(44.1)$ & $94(47.7)$ & \\
\hline 2 & $217(54.1)$ & $114(55.9)$ & $103(52.3)$ & \\
\hline Number of good quality embryos per transferred cycle, n (\%) & & & & 0.0008 \\
\hline 0 & $35(8.7)$ & $8(3.9)$ & $27(13.7)$ & \\
\hline 1 & 199 (49.6) & $100(49.0)$ & $100(50.8)$ & \\
\hline 2 & $167(41.7)$ & $96(47.1)$ & $70(36.5)$ & \\
\hline good-quality embryo rate (\%) & $87.8 \pm 0.3$ & $93.4 \pm 0.2$ & $82.0 \pm 0.4$ & 0.0001 \\
\hline Types of embryos transferred, n (\%) & & & & 0.64 \\
\hline Cleavage embryos & $190(47.4)$ & $99(48.5)$ & $91(46.2)$ & \\
\hline Blastocysts & $211(52.6)$ & $105(51.5)$ & $106(53.8)$ & \\
\hline Serum estradiol level on the day of embryo transfer (pg/ml), mean \pm s.d. & $434.8 \pm 444.5$ & $393.9 \pm 385.6$ & $477.1 \pm 495.6$ & 0.12 \\
\hline $\begin{array}{l}\text { Serum progesterone level on the day of embryo transfer }(\mathrm{ng} / \mathrm{ml}) \text {, mean } \pm \\
\text { s.d. }\end{array}$ & $15.8 \pm 7.6$ & $15.8 \pm 7.4$ & $15.8 \pm 7.8$ & 0.95 \\
\hline Endometrial thickness $(\mathrm{mm})$, mean \pm s.d. & $9.3 \pm 1.7$ & $9.5 \pm 1.7$ & $9.0 \pm 1.6$ & 0.001 \\
\hline Endometrial morphology, n (\%) & & & & 0.47 \\
\hline Type A & $345(86.0)$ & $178(87.3)$ & $167(84.8)$ & \\
\hline Type B & $56(14.0)$ & $26(12.8)$ & $30(15.2)$ & \\
\hline
\end{tabular}

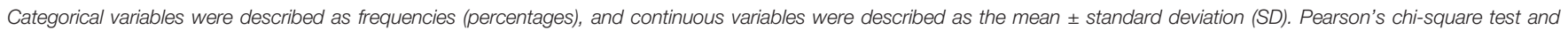
Student's t-test were used for parametric comparisons. $P$ value $<0.05$ was considered to indicate statistical significance.

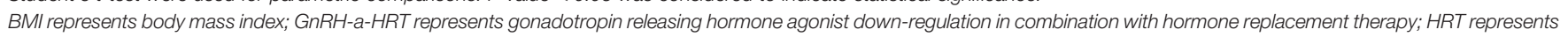
conventional hormone replacement therapy.

Grade IV: the blastomeres are seriously uneven or irregular, and the cytoplasm has serious granule phenomenon, debris $>50 \%$. Grade I and II embryos with 6-10 blastomeres on day 3 were defined as good-quality embryos.

Blastocyst score: The blastocysts were graded according to Gardner's blastocyst grading method (12). First, according to the expansion and hatching degree of the blastocysts, the blastocysts were divided into 1-6 grades: Grade 1, early blastocyst, the volume of the blastocyst cavity is less than half of the total volume of the blastocyst; Grade 2, the volume of the blastocyst cavity is more than half of the total volume of the blastocyst; Grade 3, completely expanded blastocyst, the blastocyst cavity occupies the entire blastocyst; Grade 4, after expansion, the volume of the blastocyst cavity is significantly larger than that of the early blastocyst, and the zona pellucida is thinner; Grade 5, the blastocysts hatched from the zona pellucida with lacerations; Grade 6, the blastocysts hatched completely out of the zona pellucida. Grade 3-6 blastocysts need to be scored for their inner cell mass and trophoblast cells. Inner cell mass (ICM) score: Grade A, the number of cells is large, and there are cells around the blastocyst; Grade B, the number of cells is small, and the combination is loose; Grade C, the number of cells is very small. Trophoblast ectoderm (TE) score: Grade A, with more cells and cells distributed around the blastocyst; Grade B, with fewer cells and loose epithelial cells; Grade $C$, with few cells. Blastocysts with day 5 scores $\geq 3 \mathrm{AA}, 3 \mathrm{AB}, 3 \mathrm{BA}, 3 \mathrm{BB}$ or day $6-7$ scores $\geq 4 \mathrm{AA}$, $4 \mathrm{AB}, 4 \mathrm{BA}$, and $4 \mathrm{BB}$ were regarded as good-quality blastocysts and cryopreserved as embryos. The embryos transferred included cleavage embryos and blastocysts without preimplantation genetic testing (PGT).

\section{Evaluation Index}

The main outcome was clinical pregnancy. The success of clinical pregnancy was defined as a gestational sac and an active fetal heartbeat on transvaginal ultrasound 4-5 weeks after ET.

\section{The Relationship Between Clinical Pregnancy and Other Variables}

To explore the importance of different factors in the models, we analyzed the correlations among the parameters (Pearson correlation), including age, BMI, E2, P, EMT and GQR. We compared the relationship between the successful group and the failed group.

\section{Model Establishment}

Age, BMI, EMT, GQR, E2, P and type of infertility were chosen as features and clinical pregnancy was used as a prediction result. We used four machine learning techniques, including LR, conditional inference tree, RF, and SVM, to develop fast and automated prediction models. All of the algorithms were implemented in the R (x64 4.0.4) language. In this study, 70\% of the samples were randomly selected as the training set, and the remaining 30\% were selected as the test set. Brief introductions to each classifier are given below. 


\section{Logistic Regression (LR)}

Logistic regression (LR) is a generalized linear model that can predict binary output according to a group of numerical variables. The basic function $\operatorname{glm}()$ in $\mathrm{R}$ language can be used to fit the LR model. The probability transformation formula of LR is as follows:

$$
p=\frac{1}{1+e^{-\left(\theta_{0}+\theta_{1} X_{1}+\theta_{2} X_{2}+\cdots+\theta_{n} X n\right)}}
$$

$\mathrm{Xi}$ is the eigenvalue, $\theta_{\mathrm{i}}$ is the regression efficiency, and $\mathrm{P}$ is the probability. When $\mathrm{P} \geq 0.5$ and $\mathrm{P}<0.5$, they are classified into two different categories. LR is the most effective linear classification model and it is simple, intuitive and interpretable. It is the most commonly used model in clinical data analysis.

First, the results of success or failure were used as response variables, and clinical features were used as prediction variables. The training set data were used to construct the LR model, and the coefficients in the model were given. The model based on the training set was used to classify the data of the test set and the logarithmic probability of success was output. Finally, a cross table was used to compare the prediction with the actual situation.

\section{Support Vector Machine (SVM)}

In machine learning, support vector machine (SVM) is one of the methods with a complete theory and good classification effect. SVM has strong generalization ability in small sample size, nonlinear, and high-dimensional pattern recognition problems, and good application prospects in the medical field.

The tune.svm () function in $\mathrm{R}$ language set a candidate range for each parameter. A more efficient model was generated and the performance of each parameter combination was output. Eight different gamma (from 0.000001 to 10 ) and 21 cost parameters (from 0.01 to 1010$)$ were tried. In general, $168(8 \mathrm{x}$ 21) models were fitted and the results were compared. Based on this parameter combination, we use a new SVM model to predict the sample units of the test set and give the number of errors to select the best parameter combination. Finally, the best parameter combination model is used to classify the test set. The logarithmic probability of success is output. Finally, a cross table is used to compare the prediction with the actual situation.

SVM is a very popular model which has a wide range of applications. It can be applied to the problem that the number of variables is far more than the number of sample units. This kind of problem is very common in the biomedical industry. However, one of the disadvantages of SVM is that it is difficult to understand and express the classification criteria. It is essentially a black box, and it is not as good as RF in modeling when there are many samples.

\section{Decision Tree (DT)}

Decision tree (DT) is a basic classification method. The DT model is a tree structure, which presents the process instances that are classified based on features. It includes traditional decision trees and conditional inference trees. Constructing a DT involves three main steps: feature selection, decision tree generation and decision tree pruning. 1) Feature selection is mainly determined by the degree of information change (i.e., information gain). 2) The generation of a decision tree refers to the classification of purity and homogeneity. The rpart() function in $\mathrm{R}$ language can be used to construct a decision tree. 3) Pruning: the purpose is to make the tree simpler to achieve better generalization ability. The prune() function in $\mathrm{R}$ language can be used to prune the decision tree.

The conditional inference tree is a variant of the traditional inference tree. The data are divided into two groups according to a variable. The permutation test is used to calculate the P-value of the two groups, and the variable with the minimum $\mathrm{P}$-value is selected as the grouping node. This method is repeated for each subgroup until all of the separations are not significant or the minimum node has been reached.

The conditional inference tree was obtained using the ctree() function in the Party package of the $\mathrm{R}$ language. Then, the conditional inference tree was used to classify the test set and the logarithmic probability of success was output. Finally, a cross table was used to compare the prediction with the actual situation.

\section{Random Forest (RF)}

Random forest $(\mathrm{RF})$ is a classifier that uses multiple trees to train and predict samples. Svetnik et al. generated multiple prediction models and summarized the results of the models to improve the classification accuracy in RF (13). The RF algorithm involves sampling sample units and variables to generate a large number of decision trees. From an intuitive point of view, each decision tree is a classifier (assuming that it is aimed at the classification problem); then, for an input sample, $n$ trees will have $n$ classification results. The RF integrates all of the voting results, and the category with the most voting times is designated as the final output.

Each tree is generated according to the following rules: 1. If the size of the training set is $\mathrm{N}, \mathrm{N}$ training samples are randomly extracted from the training set and put back (this sampling method is called the bootstrap sample method) as the training set of the tree. The training set of each tree is different, and it contains repeated training samples. The training set of each tree and the final classification of trained tree are the same if there is no random sampling. If it is not sampling with return, the training samples of each tree are different and there will be no intersection. Therefore, each tree is "biased" and absolutely "onesided". 2. Every tree grows as much as possible, and there is no pruning process.

The $R F()$ function in the RF package of $R$ language was used to generate the RF. The RF package is a RF generated from a traditional decision tree. Then, the RF was used to classify the test set. The logarithmic probability of success was output. Finally, a cross table was used to compare the prediction with the actual situation.

\section{Algorithm Evaluation}

After using each algorithm to train and test the data set, the performance of each algorithm is evaluated by different 
indicators, including specificity, sensitivity, positive/negative predictive rate, overall prediction accuracy and area under curve (AUC). The positive/negative predictive rate, which refers to the possibility of success/failure if the model predicts success/failure. The AUC reflects the accuracy of the algorithm.

\section{Statistical Analysis}

Categorical variables were described as frequencies (percentages), and continuous variables were described as the mean \pm standard deviation (SD). Pearson's chi-square test and Student's t-test were used for parametric comparisons. $P$ value $<$ 0.05 was considered to indicate statistical significance. All statistical analyses were performed using SPSS version 17 (SPSS Inc., Chicago, IL, USA).

\section{Human Ethics}

This study was approved by the Medical Research Ethics Committee of The First Affiliated Hospital of USTC (Approve ID: 2021-RE-062).

\section{RESULTS}

\section{Clinical Data}

We divided the female patients into the clinical pregnant and non-clinical pregnant groups. The clinical characters of the patients are summarized in Table 1. There was no significant difference between the two groups regarding some baseline characteristics (e.g., BMI, endometrial preparation protocol, number of transferred embryos per transferred cycle, number of good-quality embryos per transferred cycle, type of embryos transferred, serum estradiol level on the day of ET, serum progesterone level on the day of ET and endometrial morphology) except for age, EMT, GQR and type of infertility. Age was significantly lower in women who achieved a successful clinical pregnancy (30.7 vs. 32.7 years, $P=0.0002)$ compared to those who did not. The EMT of clinical pregnancy group was significantly thicker than that of non-clinical pregnancy group (9.5 vs. $9.0 \mathrm{~mm}, P=0.001$ ). The GQR and primary infertility rate in clinical pregnancy group were significantly higher than that in non-clinical pregnancy group $(93.4 \%$ vs. $82.0 \%, P=0.0001$; $57.4 \%$ vs. $44.7 \%, P=0.01$ ), respectively.

\section{The Relationship Between the Outcome of ET and the Factors}

The clinical characters of 401 female patients who experienced hormone replacement FET cycles were analyzed in this study. The correlations among the characters are shown in Figure 1. No correlation was found between each two clinical characters (Figure 1). Figure 2 showed that features were compared between the two groups. There were significant differences between the two groups in age, EMT and GQR (Figure 2).

\section{Evaluation of the Algorithms}

We compared the performance of these four machine learning algorithms by using accuracy, sensitivity, specificity and positive/ negative predictive rate (Table 2). Clinical characters includes age, BMI, E2, P, EMT, GQR and type of infertility. The predictive abilities of the four machine learning algorithms for clinical pregnancy were further analyzed with a receiver operating characteristic (ROC) curve (Figure 3). In addition, two variables were screen out through conditional inference tree to predict the results of FET: EMT and GQR. As shown in Figure 4, among the patients with good-quality embryos, the clinical pregnancy (success) rate of FET was approximately $70 \%$ when EMT was thicker than $9.6 \mathrm{~mm}$, but $50 \%$ when EMT was no thicker than $9.6 \mathrm{~mm}$. For the patients without good-quality embryo, the clinical pregnancy (success) rate of FET was approximately 10\% (Figure 4).

\section{DISCUSSION}

The present study adopted machine learning to help doctors make correct predictions, which are beneficial for both doctors and patients. The results show that the machine learning algorithm is suitable for the prediction of pregnancy outcome after ET, and the conditional inference tree has better prediction ability and effective predictors. The advantages of this algorithm have also been verified in other medical disciplines (14). Patients without good-quality embryos can be advised to cancel transplantation to avoid unnecessary economic losses. With the increase in medical data, machine learning algorithms have been widely used in the rapid analysis of large amounts of data. Machine learning can predict clinical outcomes by defining data attributes and using clinical data and calculation algorithms (15). It can also improve the efficiency of predicting outcomes by building different algorithms for evaluation and comparison (16). Machine learning algorithms include traditional logistic regression, support vector machines, decision trees, random forests and so on. Goyal et al. established the machine learning model to predict a successful live-birth through 30 clinical features in IVF (15). Another study identified six classification models that can accurately predict early pregnancy loss (8). Moreover, Raef et al. used 82 attributes as predictive factors to predict ET outcomes with six dominant machine learning approaches (17). These studies indicated that RF model outpace other platforms in prediction. Currently, AI is intensively researched and widely used in IVF, especially in the selection of embryos (18). The purpose of using AI in IVF embryo selection is to eliminate the potential deviation of selection based on visual evaluation alone. VerMilyea et al. established the life whisper AI model which can improve the ability of predicting embryo viability compared with the traditional embryo grading method (19). A naïve Bayes model was established to predict the pregnancy outcome of individual embryos in an IVF cycle with the aim of providing decision support on the number of embryos transferred (20). In addition, the deep learning model was able to predict clinical pregnancy from time-lapse videos with an AUC of 0.93 [95\% CI 0.92-0.94] (21). These studies demonstrated the potential of AI-based methods in improving the success rate of IVF laboratory. 

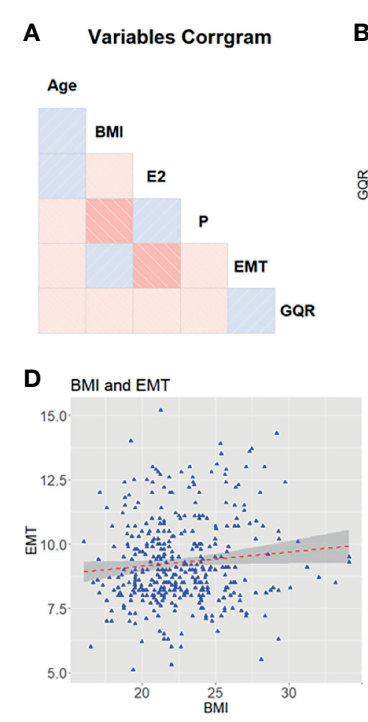
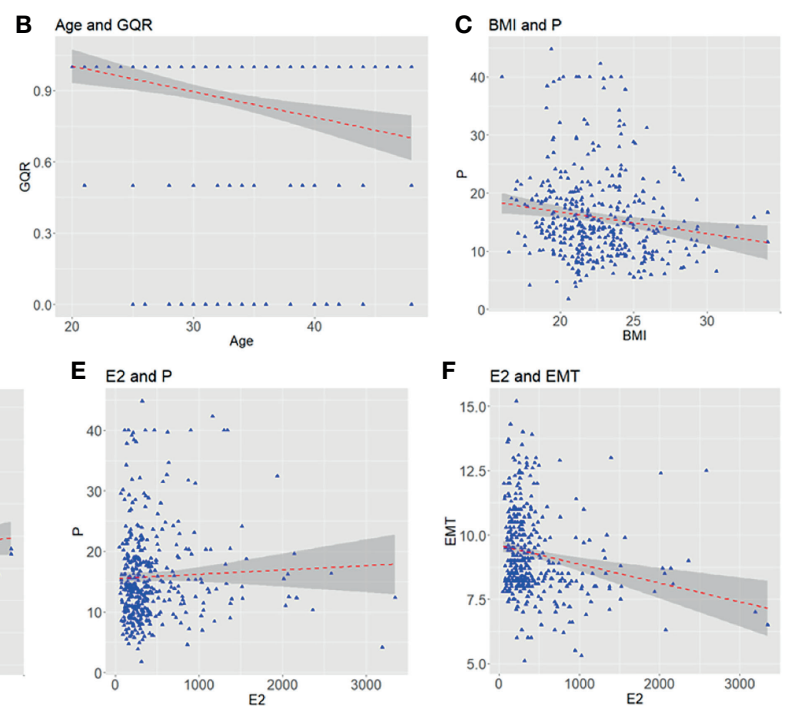

FIGURE 1 | The relationship among clinical characters (age, BMI, E2, P, EMT and GQR) from patients undergoing embryo transfer. (A) Correlation matrix of 6 features. The blue and red squares show positive correlations and negative correlations, respectively. Color depth is positively correlated with correlation coefficient. (B-F) Comparison between features. BMI, body mass index; EMT, endometrial thickness on the day of progesterone treatment; GQR, good-quality embryo rate; E2, serum estradiol level on the day of embryo transfer; P, serum progesterone level on the day of embryo transfer.

Furthermore, Yi et al. established a logistic algorithm and used FHR, GS, CRL, YSD and MA as features to predict the pregnancy outcome of 2601 ET samples (22). However, few studies have used machine learning algorithms to predict pregnancy outcomes during hormone replacement FET cycles $(9,23,24)$.

In the process of FET, there are many factors affecting the pregnancy outcome, including the age, embryo quality, endometrial receptivity and so on. A previous study showed that clinical pregnancy outcomes after FET could be accurately predicted using objective parameters, such as the woman's age (25). It has already been reported that the clinical pregnancy rate using blastocyst transfer decreases gradually with increasing maternal age, and the embryo implantation rate is significantly higher in women under the age of $35(23,26)$. In the four algorithms of the present study, age was not a strong predictor in the prediction of FET outcome. However, there were significant
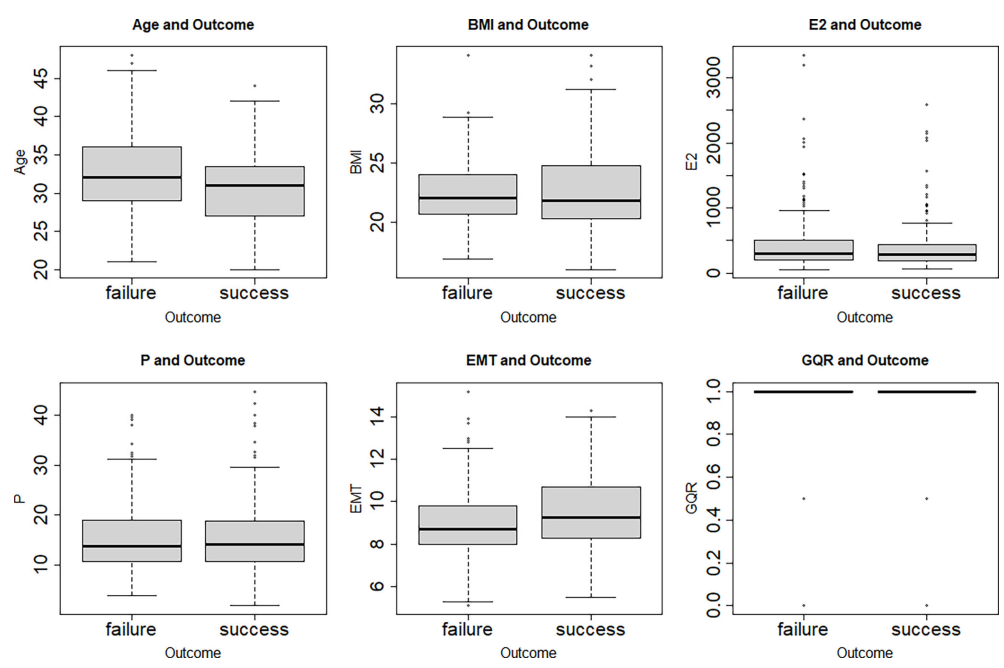

FIGURE 2 | Box plots of the age, BMI, E2, P, EMT and GQR in clinical pregnancy (success) and non-clinical pregnancy (failure) group. There were significant differences between the two groups in age $(\mathrm{t}=3.70, P=0.0002)$, EMT $(\mathrm{t}=-3.26, P=0.001)$ and $\mathrm{GQR}(\mathrm{t}=-3.84, P=0.0001)$. BMl, body mass index; EMT, endometrial thickness on the day of progesterone treatment; GQR, good-quality embryo rate; E2, serum estradiol level on the day of embryo transfer; $\mathrm{P}$, serum progesterone level on the day of embryo transfer; success: success of clinical pregnancy; failure: failure of clinical pregnancy. 
TABLE 2 | Performance comparison among the four machine learning algorithms in predicting clinical pregnancy.

\begin{tabular}{|c|c|c|c|c|c|c|}
\hline Machine learning models & AUC & Sensitivity & Specificity & Positive predictive rate & Negative predictive rate & Accuracy \\
\hline Logistic regression & 0.603 & 0.64 & 0.57 & 0.60 & 0.61 & 0.60 \\
\hline Support vector machine & 0.554 & 0.52 & 0.58 & 0.56 & 0.55 & 0.55 \\
\hline Conditional inference tree & 0.540 & 0.30 & 0.80 & 0.60 & 0.53 & 0.55 \\
\hline Random forest & 0.613 & 0.48 & 0.75 & 0.66 & 0.58 & 0.61 \\
\hline
\end{tabular}

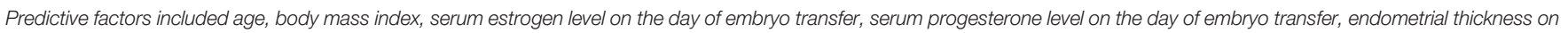
the day of progesterone treatment, good-quality embryo rate and type of infertility (primary or secondary).

differences in age and GQR between clinical pregnancy group and non-clinical pregnancy group. Age may play an indirect role by affecting the quality of oocytes. It has been reported that age and embryo quality are independent factors affecting oocyte variability and embryo implantation potential (27-31). This finding is supported by the extensive evidence in the literature of an overall adverse effect of aging on oocyte quality and hence embryo quality $(32,33)$. Studies found that age was significantly different between the primary and secondary infertility groups (34). Primary infertility refers to patients who have never had a history of pregnancy. This conclusion is consistent with the clinical characteristics of patients included in this study.

Endometrial receptivity is regulated by many factors, such as the levels of serum estrogen and progesterone and the characteristics of the endometrium. In the hormone replacement cycle, exogenous progesterone is necessary for the transformation of the endometrium from the proliferative phase to the secretory phase in the hormone replacement cycle. Recent

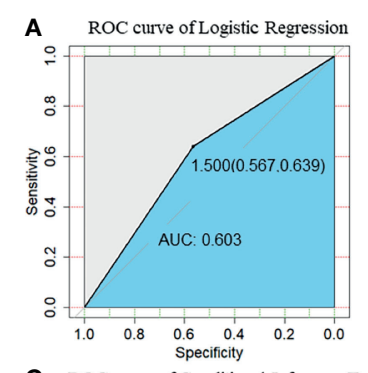

C ROC curve of Conditional Inference Tree
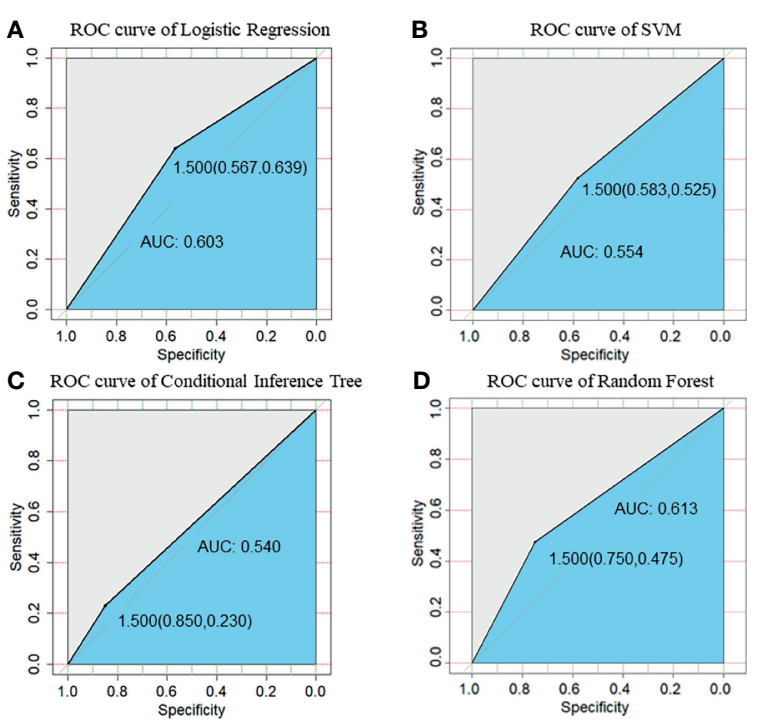

FIGURE 3 | ROC curve of prediction of clinical pregnancy with clinical characters. (A) ROC curve of Logistic Regression; (B) ROC curve of SVM; (C) ROC curve of Conditional Inference Tree; (D) ROC curve of Random Forest. Clinical characters included age, body mass index, serum estrogen level on the ET day, serum progesterone level on the ET day, endometrial thickness on the day of progesterone treatment, good-quality embryo rate and type of infertility (primary or secondary). SVM, support vector machine. studies have shown that in the early luteal phase, there is a window period for the optimal progesterone level to permit embryo implantation (35). A premature increase in progesterone or failure to reach the threshold level can lead to early closure or nonopening of the implantation window, which eventually leads to embryo implantation failure.

Studies have reported the relationship between the hormonal level on the day of ET and the pregnancy outcome in FET cycles $(36,37)$. Yovich et al. used multiple comparison analysis, which showed that the likelihood of pregnancy in FET cycles under hormonal control is highly correlated with the circulating concentration of $\mathrm{P}$ (35). Boynukalin et al. also proposed the correlation between the progesterone level on the day of ET and the persistent pregnancy rate after a hormone replacement cycle (37-39). However, these transferred embryos were euploid blastocysts. The potential influence of age and embryo quality

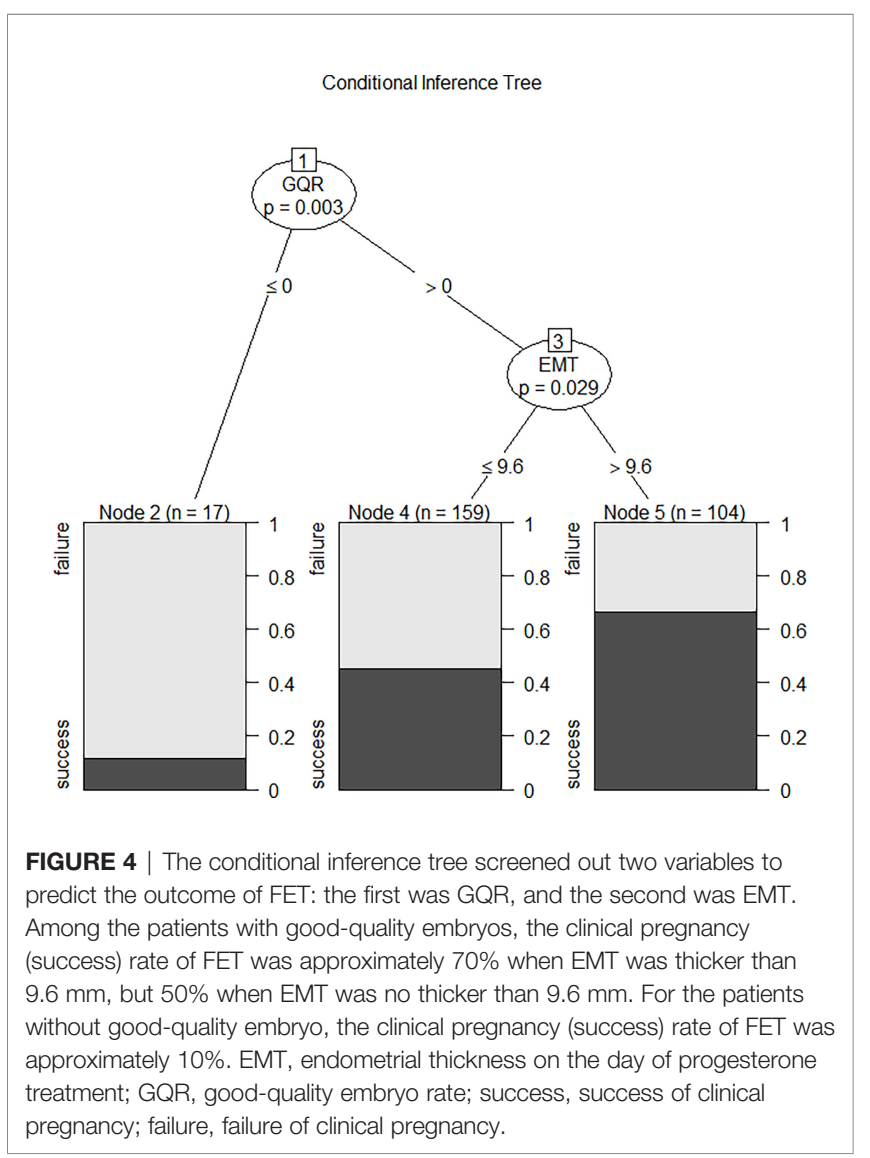


on pregnancy outcome was excluded. Niu et al. found that serum progesterone and estradiol on or before the day of ET did not predict pregnancy success in hormone replacement FET cycles $(40,41)$. The methods used in the above researches did not involve machine learning algorithms. However, we used machine learning algorithms to predict the pregnancy outcomes and concluded that serum E2 and P levels on the day of ET could not predict the pregnancy outcomes in hormone replacement FET cycles, suggesting that hormonal measurement on the day of ET in this method of endometrial preparation is unnecessary.

Richter et al. demonstrated that age, embryo quality, and EMT were related to clinical pregnancy (42). In addition to age, Michael et al. also identified EMT as an independent predictor of clinical pregnancy following blastocyst transfer. EMT thicker than $9.4 \mathrm{~mm}$ was identified as most predictive of a successful clinical pregnancy (24). In the present study, the EMT screened out by the conditional inference tree played important roles in predicting the pregnancy outcome, which is consistent with previous studies $(4,43,44)$.

There are still some limitations in this study. The prediction accuracy of the present study is limited, which suggests that there may be some other attributes that can be included as follows: 1) stimulation approach 2) oocyte quality 3) time lapse based annotations 4) male factor infertility 4) culture conditions 5) ethnic variation. In addition, the clinical data used were derived from a single center of one hospital. Therefore, more data from other centers are needed to improve the prediction performance of the algorithm.

\section{CONCLUSION}

Machine learning algorithms were used to establish four models to predict the pregnancy outcomes of patients preparing for FET with hormone replacement cycles. GQR and EMT were the predictors of clinical pregnancy screened out by conditional inference tree. This result will provide a reference for couples who are considering ET and help them make appropriate choices. This information might be useful in clinical practice because it showed there was no value in measuring E2 and P levels on the day of ET during hormone replacement cycles. In summary, these data demonstrated the potential for AI predictive models to contribute to IVF in the same way that they had contributed to other areas of human health. AI predictive models can reduce costs of assisted reproductive technology (ART) treatments by preventing repeated IVF cycles and high expenditures of ART cycles is one of the major barriers that have significant economic effects on communities especially in countries where public

\section{REFERENCES}

1. Zhang W, Xiao X, Zhang J, Wang W, Wu J, Peng L, et al. Clinical Outcomes of Frozen Embryo Versus Fresh Embryo Transfer Following In Vitro Fertilization: A Meta-Analysis of Randomized Controlled Trials. Arch Gynecol Obstet (2018) 298(2):259-72. doi: 10.1007/s00404-018-4786-5

2. Gleicher N, Kushnir VA, Sen A, Darmon SK, Weghofer A, Wu YG, et al. Definition by FSH, AMH and Embryo Numbers of Good-, Intermediate- and funding is used. In the future, we expect to improve the prediction performance of machine learning algorithms through the expansion of data sets and parameter types, hoping to find strong predictors that predict pregnancy outcomes accurately.

\section{DATA AVAILABILITY STATEMENT}

The original contributions presented in the study are included in the article/supplementary material. Further inquiries can be directed to the corresponding authors.

\section{ETHICS STATEMENT}

This study was approved by the Medical Research Ethics Committee of The First Affiliated Hospital of USTC (Approve ID: 2021-RE-062). The patients/participants provided their written informed consent to participate in this study.

\section{AUTHOR CONTRIBUTIONS}

$\mathrm{RL}$ conceived and designed the experiments, performed the experiments, analyzed the data, prepared figures, authored or reviewed drafts of the paper, approved the final draft. SB and XJ authored or reviewed drafts of the paper. LL, XT, and SZ contributed reagents/materials/analysis tools. YW and BX conceived and designed the experiments, contributed reagents/ materials/analysis tools, authored or reviewed drafts of the paper, approved the final draft. All authors contributed to the article and approved the submitted version.

\section{FUNDING}

This study was supported by National Natural Science Foundation of China (82171599, 81971333 and 81901543), and the National Key Research and Development Project (2019YFA0802600). The funders had no role in study design, data collection and analysis, decision to publish, or preparation of the manuscript.

\section{ACKNOWLEDGMENTS}

We would like thank all personnel of the Reproductive and Genetic Hospital, The First Affiliated Hospital of USTC, for their assistance in this work.

Poor-Prognosis Patients Suggests Previously Unknown IVF OutcomeDetermining Factor Associated With AMH. J Transl Med (2016) 14(1):172. doi: 10.1186/s12967-016-0924-7

3. Turner K, Reynolds-May MF, Zitek EM, Tisdale RL, Carlisle AB, Westphal LM. Stress and Anxiety Scores in First and Repeat IVF Cycles: A Pilot Study. PloS One (2013) 8(5):e63743. doi: 10.1371/journal.pone.0063743

4. Shalom-Paz E, Atia N, Atzmon Y, Hallak M, Shrim A. The Effect of Endometrial Thickness and Pattern on the Success of Frozen Embryo 
Transfer Cycles and Gestational Age Accuracy. Gynecol Endocrinol (2021) 37 (5):428-32. doi: 10.1080/09513590.2020.1821359

5. Yuval Y, Lipitz S, Dor J, Achiron R. The Relationships Between Endometrial Thickness, and Blood Flow and Pregnancy Rates in in-Vitro Fertilization. Hum Reprod (1999) 14(4):1067-71. doi: 10.1093/humrep/14.4.1067

6. Reid S, Nadim B, Bignardi T, Lu C, Martins WP, Condous G. Association Between Three-Dimensional Transvaginal Sonographic Markers and Outcome of Pregnancy of Unknown Location: A Pilot Study. Ultrasound Obstet Gynecol (2016) 48(5):650-5. doi: 10.1002/uog.15923

7. Golbasi H, Ince O, Golbasi C, Ozer M, Demir M, Yilmaz B. Effect of Progesterone/Estradiol Ratio on Pregnancy Outcome of Patients With High Trigger-Day Progesterone Levels Undergoing Gonadotropin-Releasing Hormone Antagonist Intracytoplasmic Sperm Injection Cycles: A Retrospective Cohort Study. J Obstet Gynaecol (2019) 39(2):157-63. doi: 10.1080/01443615.2018.1504204

8. Liu L, Jiao Y, Li X, Ouyang Y, Shi D. Machine Learning Algorithms to Predict Early Pregnancy Loss After In Vitro Fertilization-Embryo Transfer With Fetal Heart Rate as a Strong Predictor. Comput Methods Programs BioMed (2020) 196:105624. doi: 10.1016/j.cmpb.2020.105624

9. Xi Q, Yang Q, Wang M, Huang B, Zhang B, Li Z, et al. Individualized Embryo Selection Strategy Developed by Stacking Machine Learning Model for Better In Vitro Fertilization Outcomes: An Application Study. Reprod Biol Endocrinol (2021) 19(1):53. doi: 10.1186/s12958-021-00734-z

10. Gonen Y, Casper RF, Jacobson W, Blankier J. Endometrial Thickness and Growth During Ovarian Stimulation: A Possible Predictor of Implantation in In Vitro Fertilization. Fertil Steril (1989) 52(3):446-50. doi: 10.1016/S00150282(16)60916-0

11. Alpha Scientists in Reproductive M and Embryology ESIGo. The Istanbul Consensus Workshop on Embryo Assessment: Proceedings of an Expert Meeting. Hum Reprod (2011) 26(6):1270-83. doi: 10.1016/j.rbmo.2011.02.001

12. Gardner DK, Lane M, Stevens J, Schlenker T, Schoolcraft WB. Blastocyst Score Affects Implantation and Pregnancy Outcome: Towards a Single Blastocyst Transfer. Fertil Steril (2000) 73(6):1155-8. doi: 10.1016/S00150282(00)00518-5

13. Svetnik V, Liaw A, Tong C, Culberson JC, Sheridan RP, Feuston BP. Random Forest: A Classification and Regression Tool for Compound Classification and QSAR Modeling. J Chem Inf Comput Sci (2003) 43(6):1947-58. doi: 10.1021/ ci034160g

14. Ferre A, Poca MA, de la Calzada MD, Moncho D, Urbizu A, Romero O, et al. A Conditional Inference Tree Model for Predicting Sleep-Related Breathing Disorders in Patients With Chiari Malformation Type 1: Description and External Validation. J Clin Sleep Med (2019) 15(1):89-99. doi: 10.5664/ jcsm. 7578

15. Goyal A, Kuchana M, Ayyagari KPR. Machine Learning Predicts Live-Birth Occurrence Before in-Vitro Fertilization Treatment. Sci Rep (2020) 10 (1):20925. doi: 10.1038/s41598-020-76928-z

16. Uddin S, Khan A, Hossain ME, Moni MA. Comparing Different Supervised Machine Learning Algorithms for Disease Prediction. BMC Med Inform Decis Mak (2019) 19(1):281. doi: 10.1186/s12911-019-1004-8

17. Raef B, Maleki M, Ferdousi R. Computational Prediction of Implantation Outcome After Embryo Transfer. Health Inf J (2020) 26(3):1810-26. doi: $10.1177 / 1460458219892138$

18. Fernandez EI, Ferreira AS, Cecilio MHM, Cheles DS, de Souza RCM, Nogueira MFG, et al. Artificial Intelligence in the IVF Laboratory: Overview Through the Application of Different Types of Algorithms for the Classification of Reproductive Data. J Assist Reprod Genet (2020) 37 (10):2359-76. doi: 10.1007/s10815-020-01881-9

19. VerMilyea M, Hall JMM, Diakiw SM, Johnston A, Nguyen T, Perugini D, et al. Development of an Artificial Intelligence-Based Assessment Model for Prediction of Embryo Viability Using Static Images Captured by Optical Light Microscopy During IVF. Hum Reprod (2020) 35(4):770-84. doi: 10.1093/humrep/deaa013

20. Uyar A, Bener A, Ciray HN. Predictive Modeling of Implantation Outcome in an In Vitro Fertilization Setting: An Application of Machine Learning Methods. Med Decis Making (2015) 35(6):714-25. doi: 10.1177/ 0272989X14535984

21. Tran D, Cooke S, Illingworth PJ, Gardner DK. Deep Learning as a Predictive Tool for Fetal Heart Pregnancy Following Time-Lapse Incubation and
Blastocyst Transfer. Hum Reprod (2019) 34(6):1011-8. doi: 10.1093/ humrep/dez064

22. Yi Y, Lu G, Ouyang Y, lin G, Gong F, Li X. A Logistic Model to Predict Early Pregnancy Loss Following In Vitro Fertilization Based on 2601 Infertility Patients. Reprod Biol Endocrinol (2016) 14:15. doi: 10.1186/s12958-016-0147-z

23. Blank C, Wildeboer RR, DeCroo I, Tilleman K, Weyers B, de Sutter P, et al. Prediction of Implantation After Blastocyst Transfer in In Vitro Fertilization: A Machine-Learning Perspective. Fertil Steril (2019) 111(2):318-26. doi: 10.1016/j.fertnstert.2018.10.030

24. Traub ML, Van Arsdale A, Pal L, Jindal S, Santoro N. Endometrial Thickness, Caucasian Ethnicity, and Age Predict Clinical Pregnancy Following Fresh Blastocyst Embryo Transfer: A Retrospective Cohort. Reprod Biol Endocrinol (2009) 7:33. doi: 10.1186/1477-7827-7-33

25. Kato K, Ueno S, Yabuuchi A, Uchiyama K, Okuno T, Kobayashi T, et al. Women's Age and Embryo Developmental Speed Accurately Predict Clinical Pregnancy After Single Vitrified-Warmed Blastocyst Transfer. Reprod BioMed Online (2014) 29(4):411-6. doi: 10.1016/j.rbmo.2014.06.007

26. Goto S, Kadowaki T, Tanaka S, Hashimoto H, Kokeguchi S, Shiotani M. Prediction of Pregnancy Rate by Blastocyst Morphological Score and Age, Based on 1,488 Single Frozen-Thawed Blastocyst Transfer Cycles. Fertil Steril (2011) 95(3):948-52. doi: 10.1016/j.fertnstert.2010.06.067

27. van Loendersloot LL, van Wely M, Limpens J, Bossuyt PM, Repping S, van der Veen F. Predictive Factors in In Vitro Fertilization (IVF): A Systematic Review and Meta-Analysis. Hum Reprod Update (2010) 16(6):577-89. doi: 10.1093/ humupd/dmq015

28. Leijdekkers JA, Eijkemans MJC, van Tilborg TC, Oudshoorn SC, McLernon DJ, Bhattacharya S, et al. Predicting the Cumulative Chance of Live Birth Over Multiple Complete Cycles of In Vitro Fertilization: An External Validation Study. Hum Reprod (2018) 33(9):1684-95. doi: 10.1093/humrep/dey263

29. Yanez LZ, Han J, Behr BB, Pera RAR, Camarillo DB. Human Oocyte Developmental Potential Is Predicted by Mechanical Properties Within Hours After Fertilization. Nat Commun (2016) 7:10809. doi: 10.1038/ ncomms 10809

30. Cai Q, Wan F, Appleby D, Hu L, Zhang H. Quality of Embryos Transferred and Progesterone Levels Are the Most Important Predictors of Live Birth After Fresh Embryo Transfer: A Retrospective Cohort Study. J Assist Reprod Genet (2014) 31(2):185-94. doi: 10.1007/s10815-013-0129-4

31. Lee TH, Chen CD, Tsai YY, Chang LJ, Ho HN, Yang YS. Embryo Quality Is More Important for Younger Women Whereas Age Is More Important for Older Women With Regard to In Vitro Fertilization Outcome and Multiple Pregnancy. Fertil Steril (2006) 86(1):64-9. doi: 10.1016/j.fertnstert.2005. 11.074

32. Tatone C, Amicarelli F, Carbone MC, Monteleone P, Caserta D, Marci R, et al. Cellular and Molecular Aspects of Ovarian Follicle Ageing. Hum Reprod Update (2008) 14(2):131-42. doi: 10.1093/humupd/dmm048

33. Baird DT, Collins J, Egozcue J, Evers LH, Gianaroli L, Leridon H, et al. Fertility and Ageing. Hum Reprod Update (2005) 11(3):261-76. doi: 10.1093/humupd/ dmi006

34. Davari Tanha F, Mohseni M, Ghajarzadeh M. Sexual Function in Women With Primary and Secondary Infertility in Comparison With Controls. Int $J$ Impot Res (2014) 26(4):132-4. doi: 10.1038/ijir.2013.51

35. Yovich JL, Conceicao JL, Stanger JD, Hinchliffe PM, Keane KN. Mid-Luteal Serum Progesterone Concentrations Govern Implantation Rates for Cryopreserved Embryo Transfers Conducted Under Hormone Replacement. Reprod BioMed Online (2015) 31(2):180-91. doi: 10.1016/j.rbmo.2015.05.005

36. Brady PC, Kaser DJ, Ginsburg ES, Ashby RK, Missmer SA, Correia KF, et al. Serum Progesterone Concentration on Day of Embryo Transfer in Donor Oocyte Cycles. J Assist Reprod Genet (2014) 31(5):569-75. doi: 10.1007/ s10815-014-0199-y

37. Kofinas JD, Blakemore J, McCulloh DH, Grifo J. Serum Progesterone Levels Greater Than $20 \mathrm{Ng} / \mathrm{Dl}$ on Day of Embryo Transfer Are Associated With Lower Live Birth and Higher Pregnancy Loss Rates. J Assist Reprod Genet (2015) 32(9):1395-9. doi: 10.1007/s10815-015-0546-7

38. Boynukalin FK, Gultomruk M, Turgut E, Demir B, Findikli N, Serdarogullari M, et al. Measuring the Serum Progesterone Level on the Day of Transfer can be an Additional Tool to Maximize Ongoing Pregnancies in Single Euploid Frozen Blastocyst Transfers. Reprod Biol Endocrinol (2019) 17(1):102. doi: 10.1186/s12958-019-0549-9 
39. Labarta E, Mariani G, Holtmann N, Celada P, Remohi J, Bosch E. Low Serum Progesterone on the Day of Embryo Transfer Is Associated With a Diminished Ongoing Pregnancy Rate in Oocyte Donation Cycles After Artificial Endometrial Preparation: A Prospective Study. Hum Reprod (2017) 32(12):2437-42. doi: 10.1093/humrep/dex316

40. Niu Z, Feng Y, Sun Y, Zhang A, Zhang H. Estrogen Level Monitoring in Artificial Frozen-Thawed Embryo Transfer Cycles Using Step-Up Regime Without Pituitary Suppression: Is It Necessary? J Exp Clin Assist Reprod (2008) 5:4. doi: 10.1186/1743-1050-5-4

41. Bocca S, Real EB, Lynch S, Stadtmauer L, Beydoun H, Mayer J, et al. Impact of Serum Estradiol Levels on the Implantation Rate of Cleavage Stage Cryopreserved-Thawed Embryos Transferred in Programmed Cycles With Exogenous Hormonal Replacement. J Assist Reprod Genet (2015) 32(3):395400. doi: 10.1007/s10815-014-0402-1

42. Richter KS, Bugge KR, Bromer JG, Levy MJ. Relationship Between Endometrial Thickness and Embryo Implantation, Based on 1,294 Cycles of In Vitro Fertilization With Transfer of Two Blastocyst-Stage Embryos. Fertil Steril (2007) 87(1):53-9. doi: 10.1016/j.fertnstert.2006.05.064

43. Vaegter KK, Lakic TG, Olovsson M, Berglund L, Brodin T, Holte J. Which Factors Are Most Predictive for Live Birth After In Vitro Fertilization and Intracytoplasmic Sperm Injection (IVF/ICSI) Treatments? Analysis of 100 Prospectively Recorded Variables in 8,400 IVF/ICSI Single-Embryo Transfers. Fertil Steril (2017) 107(3):641-8.e2. doi: 10.1016/j.fertnstert.2016.12.005
44. Zhang T, Li Z, Ren X, Huang B, Zhu G, Yang W, et al. Endometrial Thickness as a Predictor of the Reproductive Outcomes in Fresh and Frozen Embryo Transfer Cycles: A Retrospective Cohort Study of 1512 IVF Cycles With Morphologically Good-Quality Blastocyst. Med (Baltimore) (2018) 97(4): e9689. doi: 10.1097/MD.0000000000009689

Conflict of Interest: The authors declare that the research was conducted in the absence of any commercial or financial relationships that could be construed as a potential conflict of interest.

Publisher's Note: All claims expressed in this article are solely those of the authors and do not necessarily represent those of their affiliated organizations, or those of the publisher, the editors and the reviewers. Any product that may be evaluated in this article, or claim that may be made by its manufacturer, is not guaranteed or endorsed by the publisher.

Copyright (c) 2021 Liu, Bai, Jiang, Luo, Tong, Zheng, Wang and Xu. This is an openaccess article distributed under the terms of the Creative Commons Attribution License (CC BY). The use, distribution or reproduction in other forums is permitted, provided the original author(s) and the copyright owner(s) are credited and that the original publication in this journal is cited, in accordance with accepted academic practice. No use, distribution or reproduction is permitted which does not comply with these terms. 